\title{
El privilegio de los pobres
}

\author{
PEDRO TRIGO \\ Centro Gumilla, Caracas, Venezuela.
}

Para mi la lectura del libro de J. Comblin, Antropologia crlstlana, ha sido una provocación. No la provocación de lo extremadamente original ni el deslumbramiento ante lo excepcionalmente brillante sino el desafío de la palabra genuinamente evangélica. La forma del libro es la proclamación. De ahí su estilo desnudo, propo. sitivo; de ahi también la seguridad vigorosa, la fuerza interior. Esto desconcierta al lector de libros de teología, acostumbrado al lenguaje técnico, a la argumentación prolija, al discurso amparado en las citas incesantes de los colegas. Frente a este discurso, científico, objetivo segün los cănones de la ciencia moderna, planta este libro su palabra directa que descansa en su propia transparencia, en su autotrascendencia, en ser la voz de un misionero, de un testigo. El autor se expone completamente $y$ por eso su exposición es desafio. No se puede leer con neutralidad: 0 se acepta o se rechaza como locura peligrosa. El libro parecerá a algunos pretencioso, arbitrario, irritante, sectario. Desde dentro alimenta y provo$\mathrm{ca}$, deja inquieto $y$ confortado, deseoso de caminar. Es lectura espiritual y a la vez teoría (comprensión de la praxis) lúcida y coherente. Este es, creo, el estatuto epistemológico del libro con sus virtualidades y con sus peligros, pero en todo caso en abierta ruptura con la literatura teológica al uso. Lo cual no significa que no dialogue con la modernidad, que una de sus caracteristicas no sea la claridad cartesiana y sobre todo que no maneje con maestría la Escritura y lo más grueso de la tradición cristiana.

Si el libro quiere decir algo concreto de parte de Dios, su mensaje puede sintetizarse así: "No hay nada mấs firme, más constante, más evi- 
dente en la antropología cristiana que el privilegio de los pobres" (48). Vamos a analizar el libro desde esta perspectiva precisa.

\section{El hombre nuevo: comunidades, mlsloneros, evangellzaclón}

El libro se abre excluyendo un enfoque: el cristianismo no ofrece una doctrina, una concepción de vida o un plan sobre el mundo, sino unos hombres y mujeres reunidos en comunidades, "el mensaje cristiano sobre la humanidad puede presentarse bajo el título paulino de 'hombre nuevo"' (19). Pues bien, "esas comunidades son Jesucristo." Revestirse del hombre nuevo es entrar en la comunidad cristiana y adoptar sus modos de vivir (23). Por eso, "sin las comunidades, la Iglesia no tendría condiciones para anunciar el hombre nuevo" (20). Ahora bien, "las comunidades nacieron y siguen estando en medio de los pobres," "ante todo en el tercer mundo" (20).

"La 'agape' es el alma de la comunidad. Una tradición burguesa reciente acostumbra a traducir agape por la palabra 'arnor' (...) es mejor traducir agape por solidaridad, que es el lazo que reúne a la comunidad" (24). "En la comunidad cristiana no hay totalidad que pueda absorber las diferencias (...) Entre las diferencias hay diălogo, y no reducción a la unidad (...) El diálogo de las diferencias es el que establece el ritmo de la convivencia" (26-27). Por eso "la comunidad representa al hombre nuevo frente a todos los individualismos y todos los totalitarismos sociales, tanto si son eclesiästicos como si son civiles o militares" (23).

Esta comunidad asi descrita "es altamente improbable," más aún "es una tarea imposible" (35). "La fe del misionero es el punto de apoyo $y$ también la única seguridad de la comunidad" (35). El, que la funda, también la vuelve a su primer fervor, discierne la novedad histórica y suscita nuevas respuestas. Y esto lo hace mediante su palabra,ésa es la única fuerza y esa fuerza radica en que trasunta a su persona. Una persona débil, sin rangos, desprotegida, enfrentada a los problemas, para que las comunidades puedan pasar por ellos con mayor seguridad. El misionero no tiene vida privada. "'Para mí la vida es Cristo' quiere decir'que mi vida está dedicada por completo a las comunidades cristianas" (31). "El misionero tiene conciencia de que él es en su vida y en su acción la encarnación y la presencia viva de Cristo y de su evangelio. El obra por lo que el misionero es" (28).

"Misionero y comunidad son correlativos y complementarios. El misionero guía a la comunidad y la comunidad guía al misionero, aunque de modo diferente" (36): "No es que ella sea instrumento de acción: jamás la comunidad será un fiel agente de ejecución del misionero; éste no tiene autoridad para exigir una obediencia estricta. La comunidad multiplica, diversifica, humaniza y respalda las inspiraciones recibidas. La salva del fanatismo o de la radicalidad, de la fuga hacia un voluntarismo abstracto."

"La evangelización de América estuvo orientada por una pléyade de misioneros de envergadura excepcional, sobre todo durante el primer siglo" (29). "En este siglo se ha renovado el carisma de misionero en América Latina con una fuerza especial (...) son el modelo concreto del hombre nuevo para nuestros tiempos" (30). Pues bien, a la larga para el misionero 
"la pobreza es el precio de su libertad y el precio de su servicio" (32).

"El hombre nuevo actúa y su acción se llama 'evangelización'"' (37).

"Evangelizar es comunicar la novedad humana: los evangelizadores son las comunidades y los misioneros que muestran en si mismos la llegada de un hombre nuevo" (39). "El evangelio es el grito y el clamor de los pueblos oprimidos (...) Ese clamor no expresa solamente la angustia de la cruz, la llamada del desamparado, sino también la confianza en la respuesta del Padre" (40).

Hasta aqui la novedad y sus sujetos. Pero ni esos sujetos son totalmente nuevos ni la novedad se da sólo en la Iglesia. Además de las C.E.B., estăn todas las nuevas estructuras y formas de colaboración que van creando los procesos de liberación. Además de los apóstoles que actúan en la Iglesia hay muchas más personas comprometidas en medio del mundo. Además de las palabras y signos eclesiales se da la acción pública de los que se empeñan en renovar hombres y estructuras (44). Por otra parte "todos los caminos siguen siendo ambiguos" (46). "Ni las instituciones más sagradas de la Iglesia se escapan de esta regla" (48).

Ahora bien, dentro de esta ambigüedad insuperable, hay que reconocer el privilegio de los pobres: "los pobres no son privilegiados porque la Iglesia los escoja, sino que la Iglesia los escoge porque Dios los ha puesto al frente del movimiento de liberación de la humanidad entera" (48). Más aún: "El cristianismo es la Iglesia de los pobres y no tiene más luces ni más fuerza que los pobres. Cuando tiene otra fuerza, se trata de una fuerza que ha pedido prestada a otros, a los poderosos de la historia" (51).

El papel de los pobres en la liberación del hombre nuevo se daria ante todo en su clamor que obliga a innovar, desestabilizando órdenes caducos. Además en las expresiones de fraternidad que surgen en ellos. También en la memoria que guardan de viejos intentos de vida comunitaria. Finalmente en los movimientos que con ayuda de otros sectores sociales logran poco a poco articular.

¿Cuál sería hoy el mensaje cristiano sobre la humanidad? Que es posible una inversión de la historia, así sea fragmentaria $e$ incompleta. Aparentemente la historia no sirve para la liberación de los pobres. Nosotros decimos, apostamos: "los pobres pueden hacer su irrupción en la historia" (54). En el tercer mundo, esta confianza en la transformación de los pobres es una novedad. Pero esto no va a ocurrir de un modo mágico. De ahi otra apuesta: "Los pobres pueden encontrar fuerzas aliadas" (54).

Puesto que en este proceso son indispensables las mediaciones históricas, se impone la necesidad del discernimiento. Muchas son las instancias que disciernen los signos de los tiempos; en definitiva el "responsable es solamente el propio pueblo de los pobres" (55). Y los criterios irían en la línea de "la mayor participación de las clases populares a partir de sus iniciativas" (56).

\section{El grito signlflcado y acontecimlen- to}

La clave del razonamiento de Comblin sería ese grito de los pobres. Si se lo interpreta de un modo voluntarista o prometeico si estariamos an- 
te una ideologización que, además de no hacer justicia a la realidad, vacía la realidad cristiana. Pero si de algo peca Comblin es de pesimismo respecto a la posibilidad del pueblo para tomar el poder y gerenciarlo. El cree por el contrario que a lo más que puede llegar el pueblo es a decir no a la situación que lo niega (ése es el sentido del grito) y a organizarse para presentar sus reivindicaciones y exigir su cumplimiento. También pueden transformar sus vidas y sus espacios de convivencia. Pero no les toca cargar con la totalidad. "Corresponde a los entendidos la tarea de elaborar una alternativa aceptable teniendo en cuenta las reinvindicaciones de los pobres" (221).

Así pues para Comblin haber recibido el evangelio y constituirse en evangelio consiste en que el pueblo pase de la resignación habitual y las esporádicas revueltas de la desesperación a la libertad de gritar consistentemente su no a la situación que lo niega y constituir espacios de participación y comunión que instauren eficazmente un diálogo histórico con otras fuerzas sociales. "La palabra de los oprimidos no presenta ninguna alternativa por si misma" (220). Y tomar la palabra es la obra de Dios en ellos. Esta palabra "es la más débil de las fuerzas humanas." El evangelio "no invade con su fuerza sino con su debilidad (...) Por eso mismo sólo en la pobreza es posible ser evangelizador" (41). Pero esta palabra es una llamada a la conversión, suscita sacrificios y entrega. Esa es su fuerza soberana.

Pues bien, Comblin escribe su Antropologia desde la novedad histórica de la irrupción de los pobres, en América Latina en buena medida a través de la interpelación explícita del evangelio de Jesús. La buena noticia es el surgimiento de este hombre nuevo. Desde ella desarrolla el autor los demás capítulos.

\section{La persona y sus derechos}

Al tratar de la persona asienta: "más importante que explicar lo que es una persona es decir quién merece el título $y$ el valor de persona humana" (69). Al comienzo de la conquista, cuando el modelo que se impone es el del conquistador, "el mensaje sobre la persona humana es éste: 'los indios son hombres"' (69). "ACtualmente, la situación de la sociedad latinoamericana sigue siendo fundamentalmente la misma" (70). Por eso sigue siendo el mismo el papel de la teología de la persona: no, definir principios morales sin entrar en particularidades, sino afirmar los derechos de personas concretas, de las personas a las que en la práctica no se reconoce' ningún derecho, es decir aceptar públicamente a los negados por el orden establecido. Porque "los derechos humanos son ante todo los derechos de los pobres (...) Defender los derechos humanos consiste en afirmar püblicamente que las leyes valen también para los pobres, que los pobres también tienen derechos" (73). Así pues, "la persona está en el rostro de los pobres," como dijo Puebla (32-39); está ahi escondida para que la descubramos (74).

\section{El cuerpo}

De esta consideración de la persona se pasa a la de su cuerpo porque "la defensa de los derechos humanos ha sido y todavía es en primer lugar la defensa de los cuerpos humillados, destruidos" (84). Asī, en contra del dualismo antiguo y moder- 
no, el cristianismo se ha edificado en torno a la encarnación del Hijo de Dios, la resurrección de la carne y las obras de misericordia corporales. Sin embargo, la institución eclesiástica, al afincarse sobre una clerecía célibe $y$ aparatada del trabajo manual, despreció y culpabilizó al cuerpo y tendió al idealismo. A él tiende también el cıentismo ya que, al objetivar el cuerpo y reducirlo a esa consideración objetual, ofrece fundamento a todas las formas de manipulación: por la medicina, la genética, ideologias, sistemas educativos y de comunicación de masas...

\section{La sexualidad}

Esta manipulación llega al colmo en el área de la sexualidad. Los antiguos no sabian explicarla científicamente, pero si sabian los estragos que la sexualidad es capaz de producir; por eso la encuadraron dentro de instituciones rígidas cuyo fin era asegurar los fines comunes: la supervivencia de la sociedad. Sabian que la permisividad lleva al suicidio. Es lo que, según el autor, estă suce diendo: "la raza blanca está en vías de rápida extinción y las naciones industrializadas de Europa y América del Norte corren el riesgo de desaparecer dentro de pocas décadas. Sin duda, la decadencia de todas las costumbres sexuales tradicionales ha sido el factor determinante que ha llevado a esta situación" (95).

Desde esta perspectiva encara la posición de la jerarquía eclesiástica ante la sexualidad. Le reprocha querer imponer la ética evangélica por medios políticos y sociales, y la intransigencia en casos límites. Pero defiende la interpretación eclesiástica de la ética evangélica. Ella no se entiende si no se la encuadra en su lucha contra el individualismo de la modernidad ya que "la reivindicación moderna de la libertad sexual está conectada con el individualismo generalizado" (106). Para la iglesia en cambio "el amor es ante todo un hecho comunitario, que está cargado de significado para la comunidad y para todo el pueblo de Dios. No se excluye el bien de la persona; pero ese bien se encuentra precisamente en aquello que une la persona con la comunidad" (106).

Para Comblin la civilización occidental "se encuentra al final de su carrera" (139), "ha resuelto desaparecer por los medios anti-conceptivos" (140). Su caida es "previsible para dentro de dos o tres décadas" (146). De ahí extrae el autor una consecuencia audaz: "la liberasción del tercer mundo, que no necesitará luchar mucho para recoger su herencia y destruir el poder de los países más desarrollados del siglo XX."

Por eso insiste que la reacción en contra de la moral sexual católica "es tipicamente burguesa" (105). "Las masas -asienta - no oponen la misma resistencia a la moral católica tradicional, ya que no la experimentan co. mo una opresión" (105). Esto no quiere decir que se adecúen a ella. No es así, pero por falta de posibilidades. "La historia de América Latina muestra dramáticamente hasta qué punto la sexualidad humana está marcada, traumatizada, deformada por el pecado social. En condiciones inhumanas de miseria material, en una situación de disgregación social o de opresión extrema, la sexualidad no consigue constituir ni la pareja equilibrada y armoniosa, ni la farmilia educadora de las generaciones futuras" (97). 


\section{Lo muerte y la vida}

Esta situación que casi impide al pueblo latinoamericano llevar una vida sexual sana constituye un pecado social porque "todo lo que destruye la vida humana es pecado. Y no hay otro pecado" (110). Y este pecado se ceba en el pueblo. "Para los pobres la muerte es una posibilidad siempre actual: hambre, sed, accidentes de trabajo, accidentes en la calle, criminalidad, cercania de las fuerzas armadas, de los policías, de los escuadrones de la muerte.... La vida se vive bajo la amenaza de la muerte, y esto condiciona la existencia. Si el peligro de muerte no es inminente, está de todos modos la amenaza de la enfermedad, de la miseria, del desempleo, de la guerra, que son formas atenuadas de la muerte porque le preparan ya el camino" (108).

Frente a esta situación el cristianismo subraya "el carácter absurdo de la muerte y su repugnancia con Dios, que es un Dios de la vida" (109); pero proclama también la victoria de Jesús sobre la muerte y se construye en torno a la fe en la vida. "La fe en Cristo no es diferente de la fa en la vida" (111). "Al contrario, Cristo viene a llevar hasta su más alta cima esa solidaridad en la fe y en la esperanza." "La modernidad occidental ha sido un cataclismo individualista del que las mayorias del mundo han sido y siguen siendo las víctimas" (114). A él le oponemos la lucha encaminada a "la organización de la convivencia" (113).

\section{La tierra}

De la consideración del cuerpo humano se pasa naturalmente a las coordenadas espaciotemporales de su existir. Ante todo la tierra. América
Latina nace como tal por la invasión del siglo XVI. Frente a las conquistas (que todavía siguen perpetrando los grandes hacendados y cuyos principales protagonistas son hoy las grandes compañías) la Iglesia proclama que la tierra y sus frutos son para todos. "De ahî la llamada 'pastoral' de la tierra"' (122).

Pero no está sólo el problema de la tierra cultivable sino de todo el planeta. La división de la humanidad tiene hoy una crasa expresión geográfica: "Literalmente la tierra tiene hoy dos caras para nuestros contemporáneos" (127). Millones de personas viven "amontonadas en medio de la suciedad. El único espectáculo que el mundo les ofrece es el de la basura provocada por la industrialización" (126). "El litoral, las montañas, los rĭos están reservados para una minoria de privilegiados. Los recursos disponibles sirven para montar una infraestructura turística en lugar de sanear las áreas de miseria" (127). Esta misma dicotomía se observa en las ciudades: "En América Latina las ciudades manifiestan la distancia entre los pueblos dominados y las oligarquías triunfantes, herederas de los conquistadores" (135). "Las comunidades cristianas son señales y vanguardias de esas grandes comunidades que tienen que ser las ciudades. Manifiestan a las ciudades su vocación" (137). "Cómo no reconocer una inspiración cristiana en la utopía muchas veces imaginada por los latinoamericanos de un mestizaje total" (132).

\section{Tlempo para vivir}

A propósito del tiempo se asienta que estamos en el tiempo de Cristo. La Pascua es el fundamento de la historia, pero también revela la ambi- 
güedad de todos los acontecimientos. Hay salvación, pues, en la historia; pero la historia no es sin más de salvación. En esta historia los pueblos y comunidades cristianos saben de la importancia de la memoria y de la ce. lebración. Pero para los cristianos el tiempo es ante todo "el tiempo de la misión de cada uno" (145). La sociedad de masas busca convertir al pueblo en espectadores ( $y$ claro está, sufridores) de la vida de los líderes $y$ las estrellas. Vivir es ver los acontecimientos políticos, militares, sociales, deportivos, incluso "intimos." Comblin insiste en que esta sensación de ver, sentir y estar en todo es pura ilusión, vida vicaria. Frente a esta manera de vivir la vida, Dios nos propone vivir personalmente en las coordenadas que nos atañen realmente, jugarnos la vida en nuestras dimensiones reales. "Alli es donde se juega toda la liberación humana" (156).

\section{La ciencla y la técnlca}

De las coordenadas del cuerpo se pasa a su constitución: la materia. Después de asentar el valor de la ciencia y de la técnica, observa Comblin desde su perspectiva: "gracias a las ciencias y tecnologias aumenta cada vez más la distancia entre países ricos $y$ pobres" (166). Ellas sirven muchas veces para justificar los privilegios de las clases dirigentes y el crecimiento del Estado" ya que dependen de capitales que sólo ellos poseen (170). Más aún "gran parte de la investigación cientifica trabaja con contratos militares." Ante esta profunda distorsión el autor se plantea "cómo hacer que los pueblos se transformen en sujetos y no en meros objetos de la ciencia y de la tecnologia" (167).

\section{El trabajo}

Pocos temas como el del trabajo dependen tanto del enfoque con que se acerque a ellos. Comblin lo hace desde la perspectiva del pobre. $Y$ ante todo constata el desprecio que aún lo rodea. "La antigua estructura de la sociedad basada en la esclavitud o en el servicio no ha desaparecido de América Latina. No se retribuye el trabajo, sino la ociosidad. Los ricos ponen su orgullo en tener el mayor número posible de empleos, esto es, de fuentes de ingresos sin trabajar" (173). En el sistema capitalista "primero es la economia, y luego los hombres que trabajan en ella (180). "Para que la economía esté subordinada a los tra. bajadores, éstos solamente pueden contar con ellos mismos" (181). "La lucha por la dignidad de los trabajadores se identificó con la lucha entre la clase de los trabajadores y la clase de los dueños de los medios de producción" (174). "Actualmente la dignidad del trabajador significa en primer lugar derecho al trabajo" (175); "lo que hace al socialismo tan atractivo para el tercer mundo es la certeza de no quedar excluido del trabajo."

"El trabajo tiene como finalidad producir comida" (180). "El evangelio biblico significa exactamente que nadie tiene derecho a comer a costa del otro" (179). "El trabajo es una tarea colectiva de la humanidad. Todos tienen que participar en ella" (180). "El mismo Jesús quiso ser trabajador. Esto quiere decir que quiso asumir su parte en la tarea común."

Desde esta perspectiva se refiere el autor al modo como la institución eclesiástica considera al trabajo. Elo. gia la Gaudlum et Spes y la Laborens Excencens, pero se pregunta 
sobre el alcance de estas declaraciones en la misma vida y estructuras de la Iglesia. La primera constatación es negativa: "los trabajadores manuales no son promovidos al sacerdocio" (178), se entiende que sin dejar de serlo. Desde ahi se pregunta por la incidencia de los trabajadores en la Iglesia. "¿Cuándo será posible que la voz de los trabajadores se exprese a través de la liturgia, de la catequesis, del estilo de las organizaciones y de las posiciones que toman las instancias representativas de la Iglesia?"

\section{Lucha del pueblo por la liberación}

"Para la teología cristiana el eje de la historia o de la vida de los hombres en sociedad es la lucha de liberación del pueblo de los pobres. En esa lucha es donde la historia recibe su significado y se vincula con la escatología, esto es, con la llegada del reino de Dios" (188).

El punto de partida es que el desarrollo por si mismo no resuelve sino que agrava los conflictos sociales (189) Esto no lo admiten todos. Por eso en América Latina necesitamos escoger entre una visión continuista y la que parte de la contradicción que nos atraviesa. Los poderosos escogen la primera. Medellin y Puebla, la segunda (191). En América Latina hay una dominación básica: "la dominaciōn de los vencedores sobre los vencidos, la de quienes tuvieron y tienen la fuerza militar y política sobre los que no tuvieron ni tienen esas fuerzas" (192). Sobre esta dominación se constituye "la dominación económica por la explotación del trabajo" (193).

"El hecho nuevo son los movimientos por los cuales los pobres se reūnen, se organizan y se constituyen como fuerzas históricas" (196). "El pueblo nace cuando empieza a tomar conciencia de la diferencia entre sus aspiraciones y las aspiraciones de la nación" (197). "Por eso mismo, las clases dominantes niegan la existencia del pueblo."

"Este pueblo es el que está surgiendo ahora en América Latina y su nacimiento constituye un hecho teológico de primera magnitud" (198). "El pueblo se manifiesta por medio de movimientos históricos: sindicatos, partidos, asociaciones locales, movimientos de liberación." "El pueblo de Dios está activo hoy en día en los esfuerzos que realiza el pueblo latinoamericano por su liberación social y política." Porque esos esfuerzos no nacen de la carne ni de la sangre. "El espiritu despierta esa fe colectiva para que un pueblo empiece a actuar como pueblo" (199). "En la medida en que son saciados los hambrientos, en que son consolados los que lloran, en que los pobres se ven levantados y los ricos humillados, la escatología ha entrado ya en este mundo" (202). Desde luego que los éxitos son precarios y los fracasos numerosos y nunca será posible encarnar en esta historia todas las aspiraciones de los pobres; sin embargo, siempre son posibles algunos cambios radicales y al contribuir a ellos se realiza no sólo la humanidad de los pobres sino la de sus aliados. Los técnicos, políticos, artis. tas y sabios. Porque "si los pobres no pueden nada por sí mismos, no dejan de ofrecer la referencia suprema para el verdadero ser humano" (203).

\section{Guerra o palabra}

En esta lucha liberadora sólo se dan dos caminos: "guerra o política, palabra o violencia" (204). "En Améri- 
ca Latina la herencia cultural idealiza hasta el extremo la guerra y las armas" (208). "Dentro de este contexto cultural, el mensaje de la acción no violenta no encuentra muchos ecos." También la Iglesia consagró frecuentemente la guerra. Pero la guerra es privilegio de los poderosos. Los pobres no tienen medios para hacer la guerra. "Jesús confirma, ratifica y al mismo tiempo fortalece una tendencia de la humanidad que fue precisamente la tendencia del pueblo de los pobres" (207). Contra él los poderosos emplearon la violencia. El no huyó. Venció el miedo a la muerte y "se enfrentó a la violencia de las armas con la fuerza de la palabra" (208). Por eso "el mensaje cristiano consiste en llevar hasta el extremo de las posibilidades el uso de la palabra" (209).

Esa palabra liberadora es el clamor de los pobres. Clamar es empezar a vencer el miedo y reivindicar el derecho a actuar en la historia como sujetos. Clamar es denunciar la mentira de la historia oficial, proclamar que ella no tiene razón. Clamar es abrir un espacio que según la ciencia de los poderosos no existe; así, pues, es descubrir la irracionalidad en que se funda la racionalidad vigente. $y$ de este modo el clamor de los pobres de. vuelve al mundo su flexibilidad. Más aún, la verdad de la ciencia y las técnicas modernas "les viene de su inserción alrededor del eje fundamental de la liberación de los pobres" (219). Ella les salva de su extravio. Igual sucede con el cristianismo: su verdad consiste en "el misterio de la liberación del pecado en que yace la huma. nidad, o, con otras palabras más actuales, la liberaciōn del pueblo de los pobres."

Pues bien, "la irrupción de los pobres en la historia latinoamericana del siglo $x \times$ es una realizaciōn ejemplar del clamor de los pobres" (215). Ellos no disponen ni de la fuerza de presión de su trabajo, sino solamente de su desnuda humanidad que se transforma en puro grito por el respeto a su dignidad. A este pueblo lo han despertado profetas: "algunos obispos, algunos sacerdotes, algunos religiosos y religiosas y millares de millares de laicos animadores de los movimientos populares" (217). Su fuerza, como dijimos, es el diălogo. "Hoy más que nunca estamos en la hora histórica del diálogo auténtico" (22).

\section{Dos Iimitaciones}

El libro se cierra con la afirmación vigorosa del aspecto individual de la liberación, y explicitando el desde dónde de las afirmaciones del libro. "Todo lo que se ha dicho hasta ahora procede directa o indirectamente, de la contemplación de Jesús y de su seguimiento" (259). Así se pone también de relieve la presencia del Espíritu de Jesús que atraviesa sus pá. ginas.

He expuesto lo que me parece ser el eje estructurador del libro y sus desarrollos más significativos. De este modo han quedado patentes los motivos por los que afirmé que el libro me había resultado provocador $y$ espiritual. Quisiera acabar señalando loqueme parecen dos limitaciones estructurales que restan dialecticidad al planteamiento. Se trata de una consideración poco compleja y dinámica del mundo de los pobres, del pueblo, y del escaso papel que juega Dios (el padre) en esta antropología cristiana (está presente como actor social, pe. ro no tanto como el tú del pueblo).

Una dimensiōn del pueblo, sin duda decisiva, es la de su pura negati- 
vidad: su privación injusta de vida acusa a la sociedad como un todo, desacraliza el orden establecido y pone al descubierto el pecado en que se funda. Cuando esta pura negatividad se atreve a expresarse (el grito) estamos ante el acto de libertad que funda la historia de liberación. Comblin señala con acierto que Dios no sólo es quien escucha el clamor de los oprimidos sino que su Espiritu es quien gime en ellos. Por eso puede ser evangelio y es el resultado de la evangelización. Pero ¿qué pasa con la historia que êl suscita? La respuesta serían las comunidades y las organizaciones populares. Pero ¿dónde está la antropología de estas múltiples formaciones sociales a medio camino entre el hombre viejo y el hombre nuevo? El capítulo I presenta la fenomenología de lo que de nuevo hay en ellas y en los misioneros que las forjan y sostienen. Pero no se señalan las concreciones graduales de esa esencia.

En las comunidades cristianas muchos de los miembros más acusiosos prosperan precisamente por serlo. Hay jövenes del pueblo que por su inquietud liberadora estudian y se gradüan en la universidad con improbo esfuerzo. Estas personas ya no son pobres: poseen vivienda con lo indispensable y trabajo estable y algo especializado. Ellas componen buena parte de las organizaciones populares. ¿Su poder será entonces el del orden establecido? En el libro se nombra ambigüedad y la procesualidad, pero las categorias de fondo son măs bien antitéticas. Por eso faltan elementos analiticos referidos a los sectores populares en cuanto se distinguen de los pobres (aunque como diferencia interna) y de los profesionales. También ésa sería la causa de que escaseen las referencias a las culturas populares, hecho llamativo tratăndose de un libro de antropología. Me parece sano huir de las vacuidades de cierto culturalismo de moda, pero se cae en el extremo opuesto. El efecto es que el pueblo, referencia principal del libro, aparezca sobre todo como ser de necesidades y como sujeto trascendental de resistencia y lucha, pero no como sujeto concreto de ellas.

La observación sobre el escaso papel de Dios tiene que ver con la anterior, en cuanto que uno de los contenidos princípales de la cultura del pueblo es la religión. En el libro Dios interviene por supuesto como agente histörico: él es el que escucha el clamor de los oprimidos y suscita liberadores. Pero no aparece como un tú en relación al cual se edifica la humanidad de las personas y señaladamente la del pueblo Es verdad que se. menciona la oración (251) como relación subjetiva, pero ella no ocupa en el libro el papel estructural que sí posee en la antropología cristiana y en la vida concreta de muchos pobres latinoamericanos. El Dios sancionador $y$ testigo (250) es fundamentalmente el de la ilustraciōn. Sí estā bien diseñado a nuestro modo de ver el papel de Jesús y de su Espiritu.

De todos modos el libro nos parece importante, más que para la lectura, para la meditación y discusión. 